\title{
The Galactic dust-to-metals ratio and metallicity using gamma-ray bursts
}

\author{
D. Watson \\ Dark Cosmology Centre, Niels Bohr Institute, University of Copenhagen, Juliane Maries Vej 30, 2100 Copenhagen Ø, Denmark \\ e-mail: darach@dark-cosmology.dk
}

Received 20 April 2011 / Accepted 21 July 2011

\begin{abstract}
The metallicity and dust-to-metals ratio of the Galaxy are fundamental parameters in understanding the interstellar medium (ISM). Currently, there is still some uncertainty surrounding these parameters. In this paper, the dust-to-metals ratio in the Galaxy is determined using the photoelectric absorption of the soft X-ray afterglows of a large sample of several hundred gamma-ray bursts (GRBs) to determine the metal column density in combination with Galactic dust maps to determine the line-of-sight dust extinction through the Galaxy in the direction of the GRB. GRB afterglows often have large extragalactic soft X-ray absorptions and therefore the GRB sample's upper-bound will define the Galactic dust-to-metals relation. Using a two-dimensional two-sample Kolmogorov-Smirnoff test, we determine this upper-bound and so derive the dust-to-metals ratio of the Galaxy. We find $N_{\mathrm{H}}=2.2_{-0.4}^{+0.3} \times 10^{21} \mathrm{~cm}^{-2} A_{V}$ asassuming solar, Anders \& Grevesse (1989, Geochim. Cosmochim. Acta, 53, 197), metallicity. This result is consistent with previous findings using bright X-ray sources in the Galaxy. Using the same technique but substituting the H I maps from the Leiden-ArgentineBonn survey for the dust maps, allows us to place a limit on the metallicity in the Galaxy. We find a metallicity consistent with the Anders \& Grevesse (1989) solar values often used in X-ray fitting. Based on this and previous studies, we suggest that the metallicity of a typical ISM sightline through the Galaxy is $\sim 0.25$ dex higher than the current best estimate of the solar metallicity. We further show that the dust-to-gas ratio seems to be correlated with the total gas column density, and that this may be due to the metallicity gradient observed toward the Galactic centre. Based on the non-constant nature of the dust-to-gas ratio, we propose that the dust column density, at $N_{\mathrm{H}}=2.2 \times 10^{21} \mathrm{~cm}^{-2} A_{V}$, represents a better proxy for the soft X-ray absorption column density than $\mathrm{H}_{\mathrm{I}}$ maps.
\end{abstract}

Key words. ISM: abundances - dust, extinction - Galaxy: abundances - X-rays: ISM - gamma-ray burst: general

\section{Introduction}

The relationship between gas, metals and dust that defines the interstellar medium (ISM), plays a central role in the properties of star-formation, and in the appearance, evolution and ultimate fate of galaxies. However the basic quantitative relationship between gas, metals and dust is still not well-defined even for our own galaxy. Many studies have been made over the years examining the dust-to-gas ratio in the Galaxy and more recently at cosmological distances. Two methods dominate these analyses: a) comparing hydrogen absorption to dust extinction or reddening, using the Ly $\alpha$ line in the UV (and sometimes $\mathrm{H}_{2} \mathrm{UV}$ lines) to get the total gas column and pairs of stars to obtain the total extinction; and b) soft X-ray photoelectric absorption that measures the total metal column density in the foreground of bright X-ray sources, converting to a gas column assuming a metallicity, and comparing this to a dust extinction obtained from methods like the Balmer decrement, the deviation in the optical/infrared from a blackbody spectrum or even measuring the dust column using the halo made by small angle scattering of X-rays off the dust. The first method provides a genuine gasto-dust ratio, in the sense that it measures the bulk of the gas directly. However, it suffers from the deficiency that it is insensitive to ionised gas and unless the molecular lines are measured, also to $\mathrm{H}_{2}$, where the fraction of hydrogen in $\mathrm{H}_{2}$ may be $\sim 0.5$ for $A_{V} \gtrsim 0.5$ (Rachford et al. 2009). Furthermore it only works along relatively low extinction lines of sight $\left(A_{V} \lesssim 2-3\right)$ since it requires spectroscopy in the UV where the extinction is far higher and stars are often too faint to observe in $\mathrm{Ly} \alpha$ or $\mathrm{H}_{2}$.
The X-ray absorption method works to very high extinctions $\left(A_{V} \sim 30\right.$ or more) and measures essentially all metals whether they are ionised or even in the solid phase, providing a census of the total column density in metals. However, it is a measurement of the metal column, not the gas column since the X-ray absorption is almost insensitive to hydrogen absorption and depends only weakly on helium. Therefore the X-ray absorption requires a metallicity conversion to move from a dust-to-metals to a dust-to-gas ratio.

It is perhaps worth noting that most studies to date in the Galaxy have either focussed on relatively nearby sets of objects or provided a small number (between 3 and about 20 for the X-ray studies) of lines of sight to locations within a few $\mathrm{kpc}$ of the Sun. Most studies in the Galaxy have therefore not probed the ISM of the Milky Way in either a complete or unbiased way. These studies have consistently found a dust-to-gas ratio at a level of $N_{\mathrm{H}} / A_{V} \sim 2 \times 10^{21} \mathrm{~cm}^{-2} \mathrm{mag}^{-1}$ (Bohlin et al. 1978; Whittet 1981; Diplas \& Savage 1994; Ryter 1996; Reina \& Tarenghi 1973; Gorenstein 1975; Ryter et al. 1975; Predehl \& Schmitt 1995; Vuong et al. 2003; Güver \& Özel 2009; Rachford et al. 2009). The statistical errors quoted for some studies have been as small as a few $10^{19} \mathrm{~cm}^{-2} \mathrm{mag}^{-1}$, however the variation from study to study is closer to a few parts in $10^{20} \mathrm{~cm}^{-2} \mathrm{mag}^{-1}$. This discrepancy may be related to an underestimate of the uncertainties or to an intrinsic scatter in the relation.

Gamma-ray bursts (GRBs), while found at cosmological distances, are extremely bright. In this paper we use the large homogeneous sample of Swift GRB X-ray afterglows to determine 
upper limits to the metal column densities to several hundred lines of sight through the Galaxy. We compare these metal column densities to all-sky hydrogen and dust surveys to obtain a new dust-to-metals ratio and metallicity value for the Galaxy. The GRB afterglows are subject to absorption by their hosts, with a minor contribution from intervening objects, so we use a 2D 2-sided Kolmogorov-Smirnov (KS) test to overcome this limitation to define the Galactic lower envelope to their absorbing column densities. Since it is X-ray selected, the sample is not afflicted by observational bias as UV studies are. However, its greatest benefit over previous samples is that this sample passes lines of sight at random through the Galaxy, and passes through the entire Galaxy in every direction, providing a set of sightlines less affected by the relatively local nature of some previous studies.

In the next section I describe the sample, data reduction and analysis techniques. In Sect. 3, I present the results of the study. Section 4 contains an analysis of the relevance of the results and a comparison to previous efforts inside and outside our Galaxy. In Sect. 5, I offer my conclusions. All errors quoted are statistical uncertainties at the $68 \%$ confidence level for one parameter of interest unless stated otherwise.

\section{Sample selection, data reduction and analysis}

The aim of the work is to obtain equivalent hydrogen column densities (in essence the metal column density, $N_{\mathrm{H}_{\mathrm{X}}}$ ) for a large number of sightlines through the Galaxy from the soft X-ray photoelectric absorption of GRB afterglows. These column densities will be a combination of the whole column density along the line of sight to the GRB, dominated by the Galactic column and the absorption from the GRB's host galaxy. This would yield a distribution of column densities with a lower limit at the values of the Galactic column density. By obtaining the Galactic dust and $\mathrm{H}_{\mathrm{I}}$ column densities $(E(B-V))$ and $N_{\mathrm{HI}}$ respectively) along the line-of-sight to the GRB from surveys, specifically Schlegel et al. (1998) for Galactic dust and Kalberla et al. (2005) for $\mathrm{H}_{\mathrm{I}}$, the dust-to-metals ratio and metallicity can be obtained by suitably fitting the two-dimensional distributions of $N_{\mathrm{H}_{\mathrm{X}}}-A_{V}$ and $N_{\mathrm{H}_{\mathrm{X}}}-N_{\mathrm{HI}}$ with a cut-off at the value of the Galactic relation. An $R_{V}=3.1$ was assumed to convert $E(B-V)$ to $A_{V}$, consistent with the mean and median $R_{V}$ values found by Fitzpatrick $\&$ Massa (2007) for 328 sources to distances up to $\sim 5 \mathrm{kpc}$ distributed across the Galactic plane.

The dust column values were obtained from Schlegel et al. (1998) and reduced by $14 \%$ following the analysis of Schlafly et al. (2010), with uncertainties based on the standard deviation of nearby points. The atomic hydrogen column densities were obtained from the Leiden-Argentine-Bonn survey reported in Kalberla et al. (2005) and accessed through the $\mathrm{nH}$ tool in Ftools. Uncertainties in the $\mathrm{H}_{\mathrm{I}}$ column density were set at $10 \%$ (Wakker et al. 2011).

To derive reddenings, Schlegel et al. (1998) use the $100 \mu \mathrm{m}$ sky emission maps from COBE/DIRBE and IRAS/ISSA with temperature corrections. The conversion from far-infrared (FIR) flux to reddening is made using the excess colours of a sample of elliptical galaxies. The zodiacal light contribution is removed using the DIRBE $25 \mu \mathrm{m}$ maps. The values of $E(B-V)$ reported should be fairly accurate, since the conversion from FIR emission is measured on reddenings, with systematic uncertainties at a level below our ultimate statistical uncertainty (Schlafly et al. 2010). The accuracy of using a conversion from reddenings to absolute extinction in the Galaxy is a matter of significant debate (e.g. Fitzpatrick \& Massa 2007), and will evidently be different along different lines of sight. However, the standard deviation found in the conversion, i.e. in $R_{V}$, is 0.27 (Fitzpatrick \& Massa 2007).

The $21 \mathrm{~cm}$ maps (Kalberla et al. 2005) are the combined Leiden/Dwingeloo (Hartmann \& Burton 1997) and Instituto Argentino de Radioastronomía (Bajaja et al. 2005) surveys detecting $\mathrm{H}_{\mathrm{I}}$ over a velocity range of $-450 \mathrm{~km} \mathrm{~s}^{-1}$ to $+400 \mathrm{~km} \mathrm{~s}^{-1}$ at $1.3 \mathrm{~km} \mathrm{~s}^{-1}$ resolution with very high equivalent main beam efficiency $(\gtrsim 0.99)$.

To obtain an equivalent hydrogen column density, $N_{\mathrm{H}_{\mathrm{X}}}$, from low-resolution X-ray spectroscopy, the deviation from a powerlaw at low energies is measured. This deviation is due to photoelectric absorption by metals (primarily $\mathrm{O}, \mathrm{C}, \mathrm{Si}, \mathrm{Fe}$ and $\mathrm{He}$ depending on the energy), and is fit with absorption models of the gas. I assume a neutral gas, although the total column density is not strongly affected by the first few ionisations of the metals since the absorption is due to inner shell electrons. Measured cross-sections are used for the elements, and while improvements to the cross-sections over the years have improved fits to absorption data (Wilms et al. 2000, WAM00), the effects on lowresolution spectroscopy are not large, a few percent at most on the total column density (WAM00). The most important effect is the assumed abundance of the elements relative to hydrogen and this is discussed in Sect. 3 below. The relative abundances of the various metals also has some effect on the total derived column density, however, at these resolutions, there is no way to distinguish which elements dominate the absorption, and since the majority of these elements contribute to the dust, it is not a crucial point.

Recent work suggests that the hot intergalactic medium (IGM) might increase the X-ray absorption smoothly with redshift at a low level (Behar et al. 2011). However 1) any such effect is small; 2) does not appear to be consistent with IGM absorption since no excess absorption is detected in about a third of quasars examined as a comparison sample; and 3) may be related to inadequately modelled Galactic absorption. Apart from smooth IGM absorption, intervening galaxies are unlikely to contribute much to the X-ray absorption in the general case as the observed absorption is related to the total metal column density and drops approximately as $(1+z)^{2.5}$ due to bandpass effects. Every line of sight would have to have the equivalent of a $0.1 Z_{\odot}$ metallicity absorber at redshift $z=1$ with a neutral gas column density of $\sim 5 \times 10^{21} \mathrm{~cm}^{-2}$ in order to begin to impact the result here. Such large column density intervening systems do not exist along most sightlines, as argued in Watson et al. (2007).

All GRBs observed with Swift's X-ray telescope (XRT) were included in the sample up to the end of November 2010 (GRB 101030A), resulting in 638 GRBs. While X-rays are detected for almost all GRBs observed by Swift-XRT, a significant fraction do not have sufficient signal-to-noise to provide a spectrum good enough to determine the X-ray absorption.

The method adopted here has been to take all pre-reduced spectra from the Swift/XRT GRB spectrum repository (Evans et al. 2009) for both the windowed timing (WT) and photon counting (PC) modes using the appropriate corresponding calibration files from that archive. These data were then fit with a power-law with photo-electric absorption (phabs (pow) in Xspec).

While the PC and WT mode spectra for a given GRB are not independent objects, they are independent measurements of the same sightline. It is well known that the absorption in the X-ray afterglows of some GRBs appears to decrease as a function of time (e.g. Starling et al. 2005; Gendre et al. 2007; Campana et al. 2007). For this reason, it is often preferable to choose the 
later PC data, as it is likely to be closer to the Galactic value. However, the WT data often has considerably higher signal. Therefore, where the PC and WT mode data gave results consistent within $1 \sigma$ (68\% confidence), both values were used. Where the results were discrepant at $>1 \sigma$, the PC value was used. Since the adopted method depends solely on the limit of the population in the $N_{\mathrm{H}_{\mathrm{X}}}-A_{V}$ plane, the result is, however, not very sensitive to this.

The cut-off, which is the relation between the X-ray column density and the $\mathrm{H}_{\mathrm{I}}$ or $A_{V}$ columns, was obtained from the data using a two-dimensional two-sample KS (2D2SKS) test. The 2D distribution was fit using a log-normal for the Galactic column density distributions (either $A_{V}$ or $N_{\mathrm{HI}}$ ) and the sum of the normalised Galactic column and an extragalactic column for the GRB afterglows, where a log-normal was also used to reproduce the extragalactic column density distribution. The parameters for the Galactic column density log-normal distribution was obtained by fitting the $A_{V}$ or $N_{\mathrm{HI}}$ distributions directly. The parameters of the extragalactic log-normal distribution as well as the normalisation of the Galactic component of the GRB afterglow absorption distribution were left as free parameters in the 2D2SKS fit. This normalisation parameter is the ratio between the X-ray absorbing column density and the $A_{V}$ or $N_{\mathrm{HI}}$. The fit was obtained by applying the 2D2SKS test at each step while stepping through each parameter across the search space, until a maximum in the probability was obtained. Uncertainties in the parameters were obtained using a Monte Carlo technique based on a Gaussian distribution of the errors on each datapoint. The same method was employed to obtain the best-fit $N_{\mathrm{HI}}-N_{\mathrm{H}_{\mathrm{X}}}$ and $A_{V}-N_{\mathrm{H}_{\mathrm{X}}}$ relations.

\section{Results}

The best-fit to the $A_{V}-N_{\mathrm{H}_{\mathrm{X}}}$ relation is $N_{\mathrm{H}}=2.2_{-0.4}^{+0.3} \times$ $10^{21} \mathrm{~cm}^{-2} A_{V}$. The best-fit for the ratio of X-ray to $21 \mathrm{~cm} \mathrm{ab}-$ sorbing column densities is $N_{\mathrm{H}_{\mathrm{X}}} / N_{\mathrm{HI}}=1.1_{-0.1}^{+0.2}$.

The results obtained are, not surprisingly, somewhat sensitive to finding a function to reproduce the distributions with reasonable fidelity. However, whether a normal or log-normal distribution is used to reproduce the extragalactic column density distribution does not affect the results significantly. This is because the fits are sensitive to where the limit of the 2D dataset is, rather than the precise shape of the distribution. As long as the distribution as a whole is reproduced with reasonable accuracy, the nature of the function is not very important.

In all of these fits the solar metallicity estimate of Anders \& Grevesse (1989, AG89) were used. It is now generally accepted that this metallicity is $\sim 45 \%$ higher than indicated by direct measurements of the solar spectrum (Asplund et al. 2009) and possibly the local ISM (WAM00). It was suggested by WAM00 that X-ray absorption studies should use among other things, updated absorption cross-sections and, in particular, updated ISM metallicity values to determine gas column densities in the Galaxy. The other improvements suggested by WAM00 have a relatively small effect on the determined gas column densities, compared to the change in metallicity they propose. Their proposed ISM abundances typically increase the equivalent hydrogen column density for a given observation by roughly the inverse of the change in the metallicity. We fitted all of our data again using the proposed absorption model of WAM00, i.e. tbabs, with abundances set at the levels suggested in that paper for the ISM. As expected, we obtain values of the $N_{\mathrm{H}_{\mathrm{X}}} / A_{V}$ and $N_{\mathrm{H}_{\mathrm{X}}} / N_{\mathrm{HI}}$ ratios that are $\sim 40 \%$ higher than using AG89 metallicities with similar fractional uncertainties. However, previous works have assumed metallicities similar to the AG89 values. Therefore it is the results using AG89 metallicities that we will use to compare with previous measurements. Indeed, as discussed further below, it appears that the metallicity of a typical Galactic sightline is not consistent with the values proposed in WAM00.

\section{Discussion}

The results presented here are consistent with the range of previous results within uncertainties. Early work focussing on bright $\mathrm{X}$-ray sources, comparing soft-X-ray metal column densities to extinction columns by Reina \& Tarenghi (1973), Gorenstein (1975), and Ryter et al. (1975) provided $N_{\mathrm{H}_{\mathrm{X}}} / A_{V}=1.9,2.2$, and $2.2 \times 10^{21} \mathrm{~cm}^{-2} \mathrm{mag}^{-1}$. Later, Predehl \& Schmitt (1995) analysed 25 point sources and four supernova remnants (SNRs) with data from ROSAT, deriving the soft X-ray absorption from the spectra of the sources. That work resulted in a value of $N_{\mathrm{H}_{\mathrm{X}}} / A_{V}=1.79 \pm 0.03 \times 10^{21} \mathrm{~cm}^{-2} \mathrm{mag}^{-1}$. To extend the sample to higher extinction lines of sight, Vuong et al. (2003) analysed the X-ray absorption and $J$-band extinction toward six nearby star-forming regions (dominated by observations of $\rho$ Oph) using pre-main sequence stars. They obtain a relation $N_{\mathrm{H}_{\mathrm{X}}} / A_{J}=5.6 \pm 0.4 \times 10^{21} \mathrm{~cm}^{-2} \mathrm{mag}^{-1}$. Converting this to $N_{\mathrm{H}_{\mathrm{X}}} / A_{V}$ using the mean Galactic extinction curve (e.g. Cardelli et al. 1989) with an $R_{V}=3.1$, yields an extremely low value, $N_{\mathrm{H}_{\mathrm{X}}} / A_{V} \sim 1 \times 10^{21} \mathrm{~cm}^{-2} \mathrm{mag}^{-1}$. In that paper the unusually low metals-to-dust ratio is ascribed to either low metallicity in the region of the Galaxy local to the Sun, or to a very flat extinction curve in the $\rho$ Oph cloud. The former hypothesis seems implausible since it requires that the gas-to-dust ratio remains constant while the metals-to-dust ratios and metallicity are lower by a factor of two. It therefore seems considerably more plausible that the dust grains in a dense molecular cloud are larger, and that therefore the extinction curve is simply flatter. Adopting $R_{V}=4$ (Vrba et al. 1993) results in $N_{\mathrm{H}_{\mathrm{X}}} / A_{V}=$ $1.7 \pm 0.1 \times 10^{21} \mathrm{~cm}^{-2} \mathrm{mag}^{-1}$; adopting $R_{V}=6$ (Vuong et al. 2003 ) gives $1.9 \times 10^{21}$ with a similar error. Observations of 38 sightlines in the far-UV allowed Rachford et al. (2009) to estimate the gas-to-dust ratio including the effects of molecularisation of the hydrogen. They found that for their sightlines, with $E(B-V)$ in the range $0.17-1.08$, the fraction of hydrogen in molecular form, $f_{\mathrm{H}_{2}}$, was typically $\sim 0.5$ with none approaching $f_{\mathrm{H}_{2}} \sim 1$. Their total gas-to-dust ratio was consistent with previous measurements, $N_{\mathrm{H}_{\mathrm{X}}} / A_{V}=2.15 \pm 0.14 \times 10^{21} \mathrm{~cm}^{-2} \mathrm{mag}^{-1}$. Finally, a recent paper by Güver \& Özel (2009) analysed this issue very systematically by selecting 22 Galactic SNRs, deriving the metal absorption from the soft X-ray spectra and the extinction from the Balmer decrement in most cases. They obtained $N_{\mathrm{H}_{\mathrm{X}}} / A_{V}=2.21 \pm 0.09 \times 10^{21} \mathrm{~cm}^{-2} \mathrm{mag}^{-1}$. In that work they suggest that while 143 SNRs exist in the Chandra, XMM-Newton, and Suzaku archives that permit good measurements of $N_{\mathrm{H}_{\mathrm{X}}}$, only the 22 they use have reasonable dust column density measurements available. An obvious extension of that work would be to obtain extinction measurements for the remaining SNRs and increase the sample size from $\sim 20$ to over a hundred. A potential improvement of that technique could be to do more than use the $\mathrm{H} \alpha / \mathrm{H} \beta$ Balmer decrement which gives a measure only of the optical reddening. If the technique could be extended to other line ratios (as recently done for GRB host galaxies, Wiersema 2011) it would significantly improve the understanding of the extinction properties of the dust columns along the line of sight.

The method used in this paper possesses several advantages over previous studies. First, the underlying X-ray source spectrum is well-defined, almost invariably dominated by a 


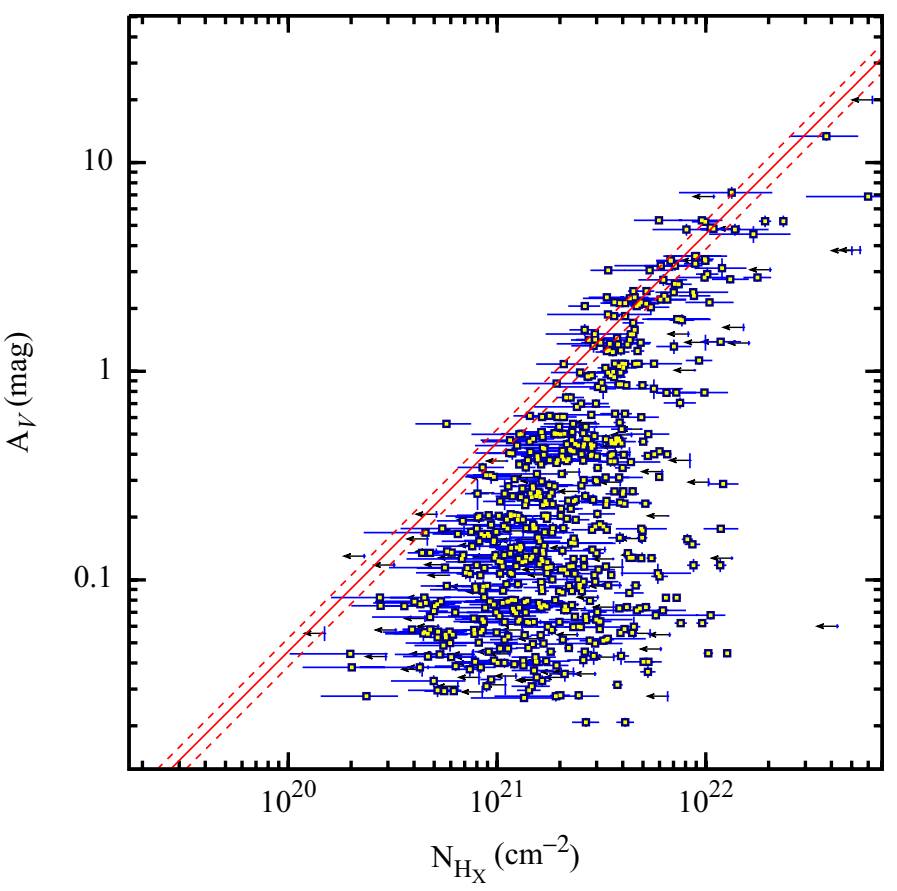

Fig. 1. The Galactic dust column $\left(A_{V}\right)$ plotted against the total equivalent hydrogen column density measured from soft X-ray absorption toward Swift GRBs $\left(N_{\mathrm{H}_{\mathrm{X}}}\right)$. The best-fit limit to the population is plotted as a solid line with $1 \sigma$ uncertainties as dashed lines and represents the Galactic relation between metals and dust: $N_{\mathrm{H}_{\mathrm{X}}} / A_{V}=2.2_{-0.3}^{+0.4} \times$ $10^{21} \mathrm{~cm}^{-2} \mathrm{mag}^{-1}$, assuming AG89 abundances to convert the metal column to an equivalent hydrogen column density.

power-law. Second, the sight-lines are passed at random through the Galaxy. Third, the sight-lines are to objects outside the Galaxy, allowing us to include the entire Galactic column in any given direction, and is therefore a good census of the whole Galaxy. However, the drawback is clearly that we are dealing with an upper-limit and therefore a large fraction of our more than 600 sightlines do not contribute much statistical power to the constraint on the metals-to-dust ratio. Partly for this reason, the uncertainties on the best-fit linear relation are fairly large, $\sim 20 \%$.

A curious fact among previous measurements deriving the metals-to-extinction ratios from X-ray data is that the quoted statistical errors are typically a few percent, while clearly the statistical quality of the fits are low, with very large outliers in terms of contributions to the fit statistic (e.g. SNR G0.0+0.0 in Güver $\&$ Özel 2009, shows a very large deviation from the fit). From this fact alone it is obvious that either the statistical or systematic uncertainties in these studies are substantially underestimated or that the intrinsic variation in the metals-to-extinction ratios is larger than the uncertainty. In addition, the studies quoted above reach mean values that differ by significantly more than a few percent, e.g. the studies of Güver \& Özel (2009) and Predehl \& Schmitt (1995) differ by more than $4 \sigma$, supporting the fact that the quoted statistical uncertainties do not represent the actual scatter in the data. The study on the $\rho$ Oph cloud is instructive in regard to deciding the origin of the scatter (Vuong et al. 2003), providing what appears to be a good quality fit for a single region in the Galaxy, and hints that perhaps it is the inherent scatter in the metals-to-extinction ratio that is responsible for the large deviation from one study to the next.

\subsection{The effect of metallicity}

The results presented here are compatible with previous observations since as far as can be determined, previous X-ray measurements used metallicity values similar to AG89 to determine the equivalent hydrogen column density. The comparison made in this paper with the $21 \mathrm{~cm} \mathrm{H}$ I measurements indicates that the mean ISM metallicity in the Galaxy is approximately the AG89 value, rather than the current best estimate of the solar metallicity as proposed by WAM00. While the uncertainties presented in this study are relatively large, a more telling comparison is with Ly $\alpha$ studies of gas-to-dust ratios in the Galaxy (Bohlin et al. 1978; Whittet 1981; Diplas \& Savage 1994, e.g.), which suggest a value of $N_{\mathrm{H} \text { I }}$ in the range $1.6-1.9 \times 10^{21} A_{V} \mathrm{~cm}^{-2}$. The ratio of the typical $\mathrm{X}$-ray-derived value to the $\operatorname{Ly} \alpha$-derived value is around 1.2, immediately indicating that the mean Galactic ISM metallicity is somewhat larger (approximately 20\%) than the solar metallicity values of AG89. Such a result is a little surprising given the debate in the X-ray literature on the correct dust-to-gas ratio. However, it has been known for many years that the ISM metallicity increases toward the Galactic centre and that the ISM metallicity becomes similar to the AG89 solar value at around $6 \mathrm{kpc}$ from the Galactic centre (Rolleston et al. 2000). Since the ratios derived in X-rays are dominated by high-extinction sightlines, it is perhaps after all not that surprising that the metallicity obtained for a typical sightline through the Galaxy is $\sim 75 \%$, or 0.25 dex, higher than the most recent measurements of the solar metallicity (Asplund et al. 2009). This value is particularly useful since it is a direct measure of the ISM itself without having to use stellar photospheres and it is effectively independent of dust depletion or ionisation in the ISM.

\subsection{Proxies for the soft $X$-ray absorbing column density}

For a typical X-ray sightline through the Galaxy, therefore, a more accurate estimate of the soft X-ray absorption may be obtained by using a metallicity value somewhat higher than that of AG89. Certainly it seems that using the current solar metallicities (Asplund et al. 2009) with H I column densities is likely to lead to a large underestimate of the Galactic soft X-ray absorption.

Since the Galaxy is known to show a strong metallicity gradient (Rolleston et al. 2000), if the dust-to-metals ratio is roughly constant (Draine 2003), one would expect the dust-to-gas ratio to vary in a similar way, i.e. a higher dust-to-gas ratio toward the Galactic centre. Sightlines toward the Galactic centre will have higher column densities: we therefore may expect a relationship in the Galaxy between the dust-to-gas ratio and the total column density. Such a relationship is possibly indicated by Schlegel et al. (1998), where the Galactic plane has a significantly higher dust-to-gas ratio than at high-latitudes with the Galactic centre direction being especially high. In Fig. 3 the relationship between the Galactic $21 \mathrm{~cm} \mathrm{H}$ I and dust column densities is shown. Sightlines toward the Galactic centre $(\cos (l) \cos (b)>0.9$, open squares in Fig. 3) do indeed seem to show a higher dust-to-gas ratio than sightlines with comparable column densities along other sightlines. The comparison of dust to gas directly yields a non-linear relation, with higher column density sightlines showing a higher dust-to-gas ratio. The linear relation $N_{\mathrm{HI}}=2.2 \times 10^{21} A_{V}$ shows clear residuals. However, $N_{\mathrm{HI}}=2.00 \pm 0.14 \times 10^{21} A_{V}^{0.77 \pm 0.07}$ provides a better fit to the data, though the fit is still not good.

Previous studies have shown a similar effect. Schlegel et al. (1998) note that the dust content of low density high velocity H I 


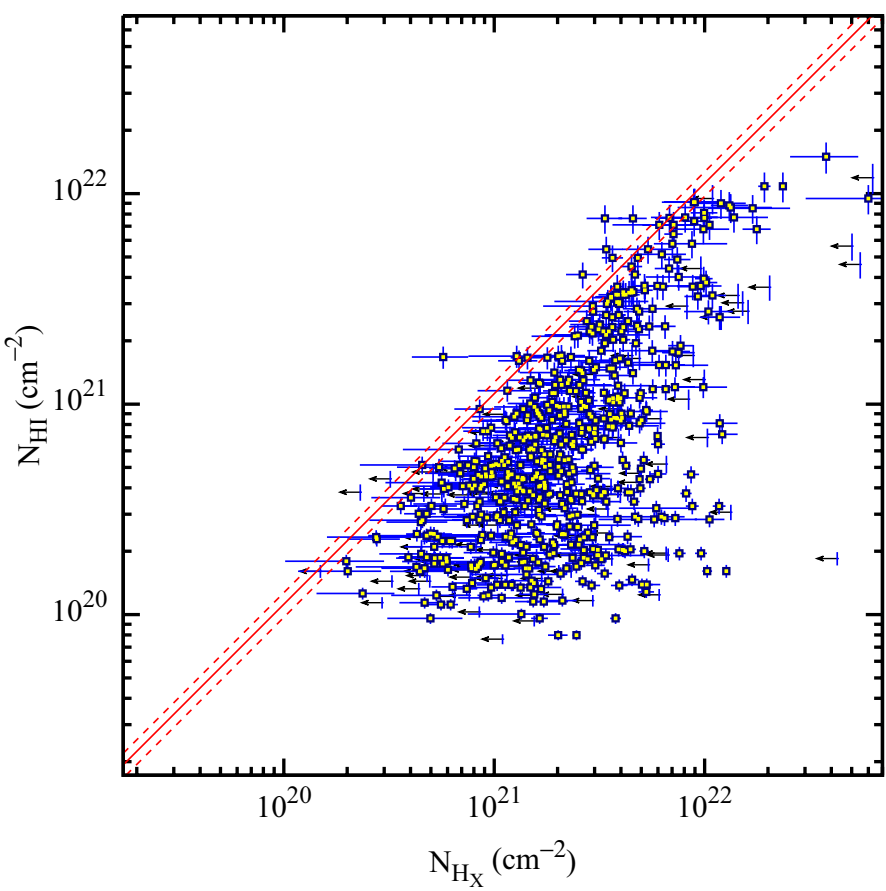

Fig. 2. The Galactic H I column density plotted against the total equivalent hydrogen column density measured from soft X-ray absorption toward Swift GRBs $\left(N_{\mathrm{H}_{\mathrm{X}}}\right)$. While the soft X-ray absorption is presented in units of equivalent hydrogen column density, it is effectively a metal column density converted to equivalent column density assuming AG89 abundances (see text). The best-fit relation is super-solar and is plotted as in Fig. 1 above. The best-fit relation, corresponding to a ratio of $N_{\mathrm{H}_{\mathrm{X}}} / N_{\mathrm{HI}}=1.1_{-0.1}^{+0.2}$, would be even higher using the current Asplund et al. (2009) solar metallicities.

clouds is lower than in other regions of the Galaxy. The survey of $\mathrm{H}$ I column densities and extinction in the UV using Ly $\alpha$ absorption and reddening of stars of Diplas \& Savage (1994) show the same effect very clearly (their Fig. 3a and to a lesser extent $3 b$ ).

In principle, a change in the absolute to selective extinction ratio, $R_{V}$, could be responsible for the apparent change in gas-to-dust ratio derived from reddening. However, since we observe the same effect in Fig. 3 where the extinction data are derived from the dust emission in the far-infrared (FIR), not from reddening, this seems unlikely. Another possibility is that dust temperatures, which strongly affect the emitted flux in the FIR, might produce such an effect - cooler lines of sight would yield a spuriously lower dust column if the assumed temperature was too high. However, Schlegel et al. (1998) accounted for the dust temperature in their analysis, and more compellingly, we observe the same effect in extinction in the UV data of Diplas \& Savage (1994).

Other explanations for the correlation of gas-to-dust ratio with column density, such as ionisation of the hydrogen at low column densities or molecularisation at high column densities, seems unlikely since the gas-to-metals ratio remains effectively constant across the range we study using $\mathrm{H}_{\mathrm{I}}$ observations (Fig. 2). It is worth noting, however, that the deviation observed at the high column end of Fig. 3 is consistent with the factor of two decrease in the Hi column density observed in far-UV molecularisation studies, where approximately half of the hydrogen exists in molecular form along sightlines with moderate or greater extinctions (Bohlin et al. 1978; Rachford et al. 2009). Even if molecularisation or ionisation were partly responsible,

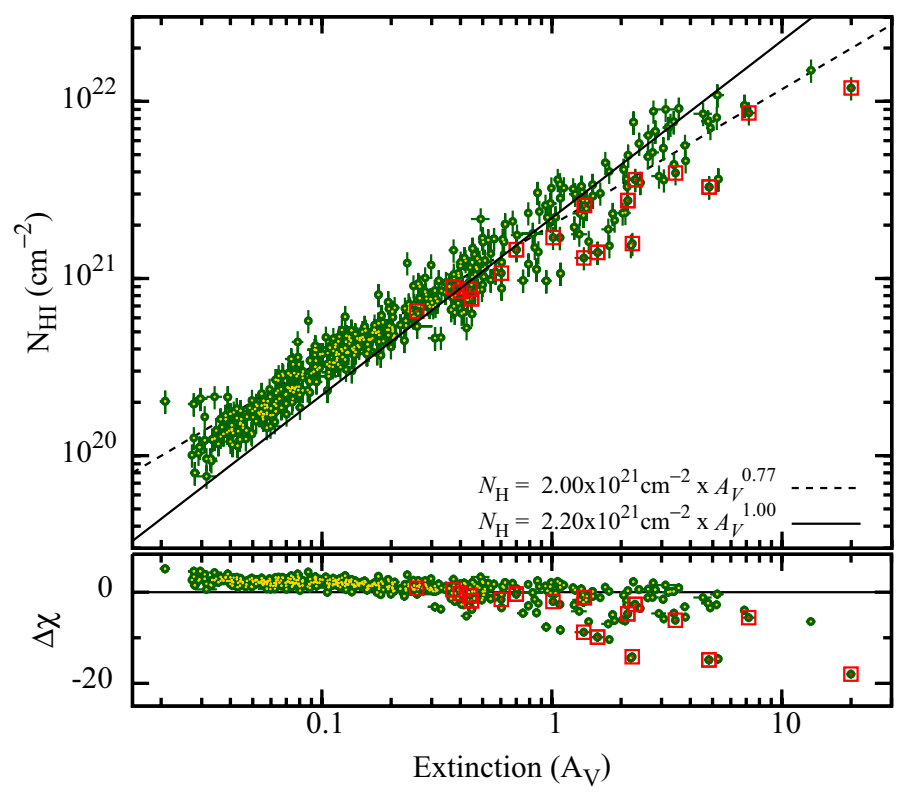

Fig. 3. Atomic hydrogen column density plotted as a function of extinction for the sightlines associated with every GRB in the sample from the LAB H i survey (Kalberla et al. 2005) and the dust maps of Schlegel et al. (1998). The anticipated linear relation is plotted as a solid line with residuals to this relation in terms of $\Delta \chi$ in the lower panel. The linear relation is clearly a poor fit to the data, suggesting that the dust-to-gas ratio increases as a function of column density. The best-fit non-linear relation is plotted as a dashed line. The non-linearity in the relation may be due to metallicity variations along different sightlines. The metallicity gradient in the Galaxy suggests that sightlines toward the Galactic centre should be more metal rich and therefore show a higher dust-togas ratio. Sightlines toward the Galactic centre $(\cos (l) \cos (b)>0.9)$ are plotted with open squares and do indeed show a higher dust-to-gas ratio than sightlines with comparable column densities.

however, it does not alter the fact that the H I column density is not the best proxy for the soft X-ray absorbing column density.

This result immediately suggests that while using $N_{\mathrm{HI}}$ as a proxy for the soft X-ray absorption is reasonable, using the dust column, as suggested by Schlegel et al. (1998), is likely to yield better results not simply because of the higher resolution of the dust maps, but because the dust-to-metals ratio seems to be more constant than the dust-to-gas ratio.

While this cannot be investigated further here due to the large uncertainty in the ratios we derive, future studies should try to determine the systematic Galactic radial and column density dependences of the dust-to-metals ratio. This latter point may be important since it is well-known that the depletion of metals out of the gas phase increases as we move into the disk and into cooler environments (Savage \& Sembach 1996), which would obviously be associated with higher column densities. It has been suggested above that the exploration of Güver \& Özel (2009) could be extended by increasing the sample size. Such an extension would indeed be worthwhile and allow the Galaxy to be divided into various lines of sight, related to the disk or the bulge. A more complete approach might be to use bright extragalactic objects known to have low host galaxy absorptions, which would allow a census to be taken of sightlines through arbitrary directions in the galaxy, including the halo. An obvious candidate are blazars, where the dust extinction could be derived either as done here from Galactic dust maps, or from reddening of the optical power-law itself (where the caveats are that the data in different bands be taken simultaneously and 
that one needs to account for intrinsic curvature of the spectra). Indeed, for a smaller sample, the X-ray and some of the opticalUV data already exists. Blazars might be bright enough for UV spectroscopy as well, allowing $\mathrm{H}_{2}$ and $\mathrm{Ly} \alpha$ measurements to be made. Such a programme, while observationally intensive for a large enough sample to probe a significant fraction of the Galaxy, could represent one of the best probes of the Galactic ISM yet devised.

\subsection{Extragalactic metals-to-dust ratios}

Studies of metals-to-dust and gas-to-dust also exist outside the local group. Metals-to-dust ratios from foreground lensing galaxies at $z \lesssim 1$ have been obtained using multiply-imaged quasars (Dai et al. 2006; Dai \& Kochanek 2009). Results show metals-to-dust ratios consistent with Galactic values. Those objects are typically relatively high mass, evolved systems. Zafar et al. (2011) showed in an analysis of GRB afterglows that GRB host galaxies, which are typically young and star-forming with low metallicities and hard radiation environments (Christensen et al. 2004; Castro Cerón et al. 2006; Savaglio et al. 2009; Svensson et al. 2010; Chary et al. 2002; Fynbo et al. 2008; Watson et al. 2010), have substantially lower dust-to-gas ratios than the local group even after accounting for metallicity.

\section{Conclusions}

An analysis of the dust-to-metals ratio and metallicity of sightlines through the Galaxy has been presented. The Galactic metal column densities were determined using the lower bound of the distribution of soft X-ray absorptions of the afterglows of a large sample of GRBs detected by the Swift satellite. The corresponding extinction and gas column densities were found using the dust and H I maps of Schlegel et al. (1998) and Kalberla et al. (2005) respectively. The metal to atomic hydrogen relation is well reproduced with a metallicity $\sim 1.75$ times the solar metallicity of Asplund et al. (2009). The best-fitting relation between metal and dust column densities is $N_{\mathrm{H}_{\mathrm{X}}} / A_{V}=$ $2.2_{-0.3}^{+0.4} \times 10^{21} \mathrm{~cm}^{-2} \mathrm{mag}^{-1}$ (using AG89 abundances). Previous observations are consistent with this result, suggesting that the metallicity for a typical ISM sightline is 0.25 dex higher than the current best value for the solar metallicity. It is therefore suggested that a better reproduction of the Galactic soft X-ray absorption will be provided with a metallicity $\sim 20 \%$ higher than the solar metallicity of AG89.

However, it is also found that a linear representation does not reproduce the gas-to-dust relationship very well. A gas-to-dust relationship with $N_{\mathrm{H} \text { I }}=2.00 \pm 0.14 \times A_{V}^{0.77 \pm 0.07}$ provides a much better fit to the data. It is very likely that this is predominantly a metallicity-gradient effect, and it is therefore concluded that while the gas-to-dust relation may be as given above, the best proxy for the Galactic soft X-ray absorption should be given by the dust column density with a relation of $N_{\mathrm{H}_{\mathrm{X}}} / A_{V}=2.2 \times$ $10^{21} \mathrm{~cm}^{-2} \mathrm{mag}^{-1}$ or $N_{\mathrm{H}_{\mathrm{X}}} / E(B-V)=6.8 \times 10^{21} \mathrm{~cm}^{-2} \mathrm{mag}^{-1}$ for an $R_{V}=3.1$.

Acknowledgements. The Dark Cosmology Centre is funded by the DNRF. I would like to thank Anja C. Andersen, Jens Hjorth, Daniele Malesani, Johan Fynbo, and Marten van Kerkwijk for useful discussions. This work made use of data supplied by the UK Swift Science Data Centre at the University of Leicester.

\section{References}

Anders, E., \& Grevesse, N. 1989, Geochim. Cosmochim. Acta, 53, 197 Asplund, M., Grevesse, N., Sauval, A. J., \& Scott, P. 2009, ARA\&A, 47, 481 Bajaja, E., Arnal, E. M., Larrarte, J. J., et al. 2005, A\&A, 440, 767 Behar, E., Dado, S., Dar, A., \& Laor, A. 2011, ApJ, 734, 26 Bohlin, R. C., Savage, B. D., \& Drake, J. F. 1978, ApJ, 224, 132 Campana, S., Lazzati, D., Ripamonti, E., et al. 2007, ApJ, 654, L17 Cardelli, J. A., Clayton, G. C., \& Mathis, J. S. 1989, ApJ, 345, 245

Castro Cerón, J. M., Michałowski, M. J., Hjorth, J., et al. 2006, ApJ, 653, L85 Chary, R., Becklin, E. E., \& Armus, L. 2002, ApJ, 566, 229

Christensen, L., Hjorth, J., \& Gorosabel, J. 2004, A\&A, 425, 913

Dai, X., \& Kochanek, C. S. 2009, ApJ, 692, 677

Dai, X., Kochanek, C. S., Chartas, G., \& Mathur, S. 2006, ApJ, 637, 53

Diplas, A., \& Savage, B. D. 1994, ApJ, 427, 274

Draine, B. T. 2003, ARA\&A, 41, 241

Evans, P. A., Beardmore, A. P., Page, K. L., et al. 2009, MNRAS, 397, 1177

Fitzpatrick, E. L., \& Massa, D. 2007, ApJ, 663, 320

Fynbo, J. P. U., Prochaska, J. X., Sommer-Larsen, J., Dessauges-Zavadsky, M., \& Møller, P. 2008, ApJ, 683, 321

Gendre, B., Galli, A., Corsi, A., et al. 2007, A\&A, 462, 565

Gorenstein, P. 1975, ApJ, 198, 95

Güver, T., \& Özel, F. 2009, MNRAS, 400, 2050

Hartmann, D., \& Burton, W. B. 1997, Atlas of Galactic Neutral Hydrogen (Cambridge, UK: Cambridge University Press)

Kalberla, P. M. W., Burton, W. B., Hartmann, D., et al. 2005, A\&A, 440, 775

Predehl, P., \& Schmitt, J. H. M. M. 1995, A\&A, 293, 889

Rachford, B. L., Snow, T. P., Destree, J. D., et al. 2009, ApJS, 180, 125

Reina, C., \& Tarenghi, M. 1973, A\&A, 26, 257

Rolleston, W. R. J., Smartt, S. J., Dufton, P. L., \& Ryans, R. S. I. 2000, A\&A, 363, 537

Ryter, C. E. 1996, Ap\&SS, 236, 285

Ryter, C., Cesarsky, C. J., \& Audouze, J. 1975, ApJ, 198, 103

Savage, B. D., \& Sembach, K. R. 1996, ARA\&A, 34, 279

Savaglio, S., Glazebrook, K., \& Le Borgne, D. 2009, ApJ, 691, 182

Schlafly, E. F., Finkbeiner, D. P., Schlegel, D. J., et al. 2010, ApJ, 725, 1175

Schlegel, D. J., Finkbeiner, D. P., \& Davis, M. 1998, ApJ, 500, 525

Starling, R. L. C., Vreeswijk, P. M., Ellison, S. L., et al. 2005, A\&A, 442, L21

Svensson, K. M., Levan, A. J., Tanvir, N. R., Fruchter, A. S., \& Strolger, L. 2010, MNRAS, 405,57

Vrba, F. J., Coyne, G. V., \& Tapia, S. 1993, AJ, 105, 1010

Vuong, M. H., Montmerle, T., Grosso, N., et al. 2003, A\&A, 408, 581

Wakker, B. P., Lockman, F. J., \& Brown, J. M. 2011, ApJ, 728, 159

Watson, D., Hjorth, J., Fynbo, J. P. U., et al. 2007, ApJ, 660, L101

Watson, D., French, J., Christensen, L., et al. 2010, ApJ, submitted [arXiv: 1010.1783]

Whittet, D. C. B. 1981, MNRAS, 196, 469

Wiersema, K. 2011, MNRAS, 414, 2793

Wilms, J., Allen, A., \& McCray, R. 2000, ApJ, 542, 914

Zafar, T., Watson, D., Fynbo, J. P. U., et al. 2011, A\&A, 532, A143 